\title{
Headache at the onset of stroke: Frequencies, background characteristics and correlation with mortality
}

\author{
Kazuo Shigematsu ${ }^{1^{*}}$, Hiromi Nakano ${ }^{2}$, Yoshiyuki Watanabe ${ }^{3}$, Tatsuyuki Sekimoto ${ }^{4}$, \\ Kouichiro Shimizu ${ }^{5}$, Akihiko Nishizawa ${ }^{6}$, Atsushi Okumura ${ }^{7}$, Masahiro Makino ${ }^{8}$, Touru Seki ${ }^{9}$, \\ Kazuhiko Bando ${ }^{10}$, Yasushi Kitagawa ${ }^{11}$ \\ ${ }^{1}$ Department of Neurology, National Hospital Organization, Minami Kyoto Hospital, Kyoto, Japan; \\ *Corresponding Author: shigemak@skyoto.hosp.go.jp \\ ${ }^{2}$ Department of Neurosurgery, Kyoto Kidugawa Hospital, Kyoto, Japan \\ ${ }^{3}$ Department of Epidemiology for Community Health and Medicine, Kyoto Prefectural University of Medicine, Graduate School of \\ Medical Science, Kyoto, Japan \\ ${ }^{4}$ Department of Neurosurgery, Kyoto Prefectural Yosanoumi Hospital, Kyoto, Japan \\ ${ }^{5}$ Department of Neurosurgery, Kyoto Fushimi Shimizu Hospital, Kyoto, Japan \\ ${ }^{6}$ Department of Internal Medicine, The Nishizawa Clinic, Kyoto, Japan \\ ${ }^{7}$ Department of Neurosurgery, Jujyo Rehabilitation Hospital, Kyoto, Japan \\ ${ }^{8}$ Department of Neurology, Japanese Red Cross Kyoto Daini Hospital, Kyoto, Japan \\ ${ }^{9}$ Department of Internal Medicine, The Seki Clinic, Kyoto, Japan \\ ${ }^{10}$ Department of Internal Medicine, The Bando Clinic, Kyoto, Japan \\ ${ }^{11}$ Department of Internal Medicine, The Kitagawa Clinic, Kyoto, Japan
}

Received 17 October 2012; revised 18 November 2012; accepted 30 November 2012

\section{ABSTRACT}

Background: Headache is a common symptom and sometimes makes patients worry about stroke. Some stroke patients actually have headache. However, the frequency of headache at the onset of stroke and the relationship between headache and outcomes are not fully clarified. Objective: The aim of the study is to clarify frequency of headache, and to compare patients with headache with patients without headache in their characteristics, risk factors and early mortalities. Methods: We confirmed the presence or absence of onset headache in 1671 patients in the Kyoto Stroke Registry. We studied frequencies of headache, age, sex, blood pressure, arrhythmia, diabetes mellitus, hyperlipemia, tobacco and alcohol use and mortality of stroke patients. Results: Headache was observed in $21.4 \%$ of stroke patients; $12.0 \%$ in cerebral infarction $(\mathrm{Cl}), 29.8 \%$ in cerebral hemorrhage $(\mathrm{CH})$, and 93.9\% in subarachnoid hemorrhage (SAH). Blood pressure at the first medical examination after stroke was higher in patients with headache. Factors associated with headache were age, diastolic blood pressure, hypertension and hyperlipemia histories, alcohol use, and paresis in $\mathrm{Cl}$, age, alcohol and tobacco use and paresis in $\mathrm{CH}$ and systolic blood pressure in SAH. The mortality rate 30 days after the stroke event was higher by $6.8 \%$ in $\mathrm{Cl}$ and by $11.4 \%$ in $\mathrm{CH}$ in patients with headache than that in patients without headache. The hazard ratios for death between patients with headache and patients without headache were 4.94 (1.73 - 14.08, $\mathrm{p}=0.003)$ in $\mathrm{Cl}$, and 3.20 (1.10 - 9.36, p = 0.033) in $\mathrm{CH}$. Conclusions: Headache is common in stroke and predicts outcome. Headache is associated with younger age and alcohol use in $\mathrm{Cl}$ and $\mathrm{CH}$. Patients who could express presence of headache at the onset of $\mathrm{Cl}$ or $\mathrm{CH}$ have higher mortality than patients who could express absence of headache. Headache is important both as an identifier of stroke and as a poor prognostic indicator in stroke.

Keywords: Headache; Stroke; Outcomes; Mortality; Epidemiology

\section{INTRODUCTION}

Headache is a common symptom in stroke and possibly correlates with the outcome [1]. Although headache is not a specific symptom of stroke [2-4], it is important both for patients and physicians. It is most often accompanied with subarachnoid hemorrhage (SAH), next with cerebral hemorrhage $(\mathrm{CH})$, and least with cerebral infarc- 
tion (CI) [5]. However, frequencies of headache among stroke subtypes are still controversial. Moreover, the relationship between headache at the onset of stroke and outcome remains unclear. Although many studies have investigated correlations between headache and other factors, such as age, sex and stroke localization, but the relationship between headache and mortality has not been fully demonstrated. Various factors have been reported as predictors of mortality [6]. The outcome after ischemic stroke depends on initial stroke severity, expressed by the NIHSS score, treatment, and age. Also, the outcome after $\mathrm{CH}$ is dependent on the initial volume of the hematoma, hematoma location, and GCS score. Nevertheless, prediction of stroke outcome in the acute stage is still difficult [7-9]. The relationship between the symptoms at the onset of a stroke and early mortality should provide valuable information to identify patients who could benefit most from intensive attention at the earliest stage of care [10,11].

The hypothesis of this study is that headache at the onset of the stroke may correlate with early mortality. To verify this hypothesis, we calculated the hazard ratios for death, comparing patients with headache and patients without headache.

\section{METHODS}

\subsection{Data Collection}

Kyoto prefecture of Japan is continuing its efforts to study various aspects of stroke events, and has been trying to collect data for all newly developed stroke patients in the entire prefecture, in cooperation with the Kyoto Medical Association and hospitals belonging to the Association. We investigated the existence or absence of a headache in stroke patients registered to Kyoto Stroke Registry (KSR) from January 1999 to December 2000 [12]. Local physicians diagnosed patients as stroke according to the WHO definition [13] and registered them to KSR with the physical findings, age, sex, histories of hypertension, arrhythmia, hyperlipemia, tobacco and alcohol use, and whether the patient was dead or alive 30 days after the admission. In cases patients died within 30 days, survival days were recorded.

Inclusion criteria: all stroke patients at any age that lived in Kyoto Prefecture, patients able to provide reliable information on the presence or absence of onset headache. Exclusion criteria: patients with particular causes; trauma, pregnancy or child birth or confinement, intoxication, neoplasm, blood disease, encephalitis, meningitis, and complication of medical interventions.

The presence, or absence, of a headache at the onset of the stroke was confirmed by the local physician based on the information from the patients and/or people who were with the patients at that time. We excluded patients unable to provide reliable information on the presence or absence of onset headache; reasons including aphasia, delirium, dementia, or depressed level of consciousness. To avoid the influence of any medication or surgical treatment, headache which developed only after interventions at the hospitals was not regarded as headache at the onset of the stroke. We did not investigate headache history that occurred more than a day before the stroke event. Systolic hypertension was defined when systolic blood pressure exceeded $140 \mathrm{mmHg}$, and diastolic hypertension was defined when diastolic blood pressure exceeded $90 \mathrm{mmHg}$. A patient's hypertension, arrhythmia and diabetes mellitus histories were diagnosed by the local physicians, based on the clinical data, based on the information given to the examining clinician by patients themselves or their family, and also based on medicines that patients have been taking. Tobacco and alcohol use were based on the information from patients and their families.

This study was approved by the board of the Kyoto Medical Association and by the Department of Health and Welfare, Kyoto Prefecture, and by the Ethics Committee of the National Hospital Organization, Minami Kyoto Hospital.

\subsection{Statistical Analysis}

The frequencies of categorical characteristics, namely headache, sex, past histories, arrhythmia, tobacco and alcohol use and paresis, among the three stroke types were determined and their differences in any two-subtypes were examined with Fisher's exact test for significance. Differences in continuous numerical variables, namely age, systolic and diastolic blood pressures among the three types of stroke patients were examined with Student's $t$ test. Kaplan-Meier curves of estimated survival were generated, and comparisons between patients with and without headache in each stroke subtype were performed using the log-rank test. The Cox proportional hazards regression was used to calculate adjusted hazard ratios and their 95\% confidence intervals for the risk of death in patients, comparing patients with and without headache. We used SPSS ver. 19 and significance was set at $\mathrm{p}<0.05$. Every reported $\mathrm{p}$ value was 2 -sided.

\section{RESULTS}

We confirmed the presence or absence of onset headache in 1671 patients. Examinations included CT ( $\mathrm{n}=$ 1470, 89.4\%), MRI scans ( $\mathrm{n}=1054,64.1 \%)$, angiography $(\mathrm{n}=429,26.1 \%)$, and scintigraphy $(\mathrm{n}=238,14.5 \%)$. Patient characteristics are summarized in Table 1.

A total of 71 out of 1603 (4.4\%) stroke patients died within 30 days of the onset of the stoke. We could not confirm if the status of 71 (4.2\%) patients was alive or 
Table 1. Characteristics of stroke patients in this study cohort.

\begin{tabular}{|c|c|c|c|c|}
\hline & $\begin{array}{r}\text { Overall } \\
\mathrm{n}=1671\end{array}$ & $\begin{array}{l}\text { Cerebral infarction } \\
\text { (CI) } n=1199\end{array}$ & $\begin{array}{l}\text { Cerebral hemorrhage } \\
(\mathrm{CH}) \mathrm{n}=325\end{array}$ & $\begin{array}{l}\text { Subarachnoid hemorrhage } \\
\text { (SAH) } n=114\end{array}$ \\
\hline Headache, \% (n = with/without) & $21.4(351 / 1314)$ & $12.0(144 / 1055)^{* 1 * 3}$ & $29.8(97 / 228)^{*_{1 * 2}}$ & $93.9(107 / 7)^{* 2 * 3}$ \\
\hline Age (SD) & 70.4 (12.3) & $71.8(11.3)^{* 1_{1 * 3}}$ & $68.9(13.2)^{* 1 * 2}$ & $61.6(14.3)^{* 2 * 3}$ \\
\hline Sex, \% female $(n=$ female/male $)$ & $44.9(750 / 921)$ & $41.0(492 / 707)^{*_{1 * 3}}$ & $52.3(170 / 155)^{*_{1} *_{2}}$ & $62.3(71 / 43)^{* 2 * 3}$ \\
\hline Systolic blood pressure (SD) (mmHg) & $161.3(30.3)$ & $159.5(29.3)^{* 1}$ & $169.7(31.9)^{*_{1} *_{2 *}^{*}}$ & $156.7(32.6)^{* 2}$ \\
\hline Diastolic blood pressure (SD) (mmHg) & $87.1(17.4)$ & $86.2(16.3)^{* 1}$ & $90.5(19.3)^{* 1}$ & $86.4(22.2)$ \\
\hline Hypertension history, \% (n = with/without) & $59.9(921 / 616)$ & $58.8(667 / 468)^{* * 3}$ & $69.8(208 / 90)^{*_{1} *_{2}}$ & $43.0(43 / 57)^{* 2 * 3}$ \\
\hline Arrhythmia, \% (n = with/without) & $14.3(232 / 1388)$ & $17.2(201 / 967)^{*_{1} * 3}$ & $7.0(22 / 294)^{*_{1}}$ & $4.8(5 / 99)^{* 3}$ \\
\hline Arrhythmia history, \% (n = with/without) & $17.9(280 / 1282)$ & $22.2(251 / 881)^{* 1 * 3}$ & $6.7(20 / 277)^{* 1}$ & $4.0(4 / 97)^{* 3}$ \\
\hline $\begin{array}{l}\text { Diabetes mellitus history, \% } \\
\text { (n = with/without) }\end{array}$ & $22.8(360 / 1219)$ & $26.5(305 / 846)^{* * 3}$ & $15.2(45 / 251)^{*_{1 * 2}}$ & $6.0(6 / 94)^{* 2 * 3}$ \\
\hline Hyperlipemia history, \% (n = with/without) & $19.0(293 / 1249)$ & $22.8(257 / 870)^{*_{1} * 3}$ & $8.3(24 / 264)^{* 1}$ & $6.1(6 / 92)^{* 3}$ \\
\hline Tobacco use, \% (n = with/without) & $31.3(563 / 823)$ & $33.4(347 / 692)^{* 1}$ & $22.2(56 / 196)^{*_{1} * 2}$ & $36.7(33 / 57)^{* 2}$ \\
\hline Alcohol use, \% (n = with/without) & $40.6(563 / 828)$ & $39.8(408 / 617)$ & $41.5(103 / 145)$ & $51.1(45 / 43)$ \\
\hline Paresis, \% (n = with/without) & $73.9(1218 / 431)$ & $78.8(939 / 253)^{* 3}$ & $78.1(250 / 70)^{* 2}$ & $14.2(15 / 91)^{* 2 * 3}$ \\
\hline
\end{tabular}

Over all includes others $(\mathrm{n}=33)$. Significant differences between CI and CH, CH and SAH, and SAH and CI were marked by ${ }^{* 1}{ }^{* 2}$, and ${ }^{* 3}$ respectively. Data on some characteristics were missing in a few patients.

dead in the cohort of the study. A total of 25 patients out of $1151(2.2 \%)$ died in cases of CI, a total of 25 patients out of 310 (8.1\%) died in $\mathrm{CH}$, and a total of 21 patients out of 111 (18.9\%) died in SAH. The mortality 30 days after the onset was significantly different among stroke subtypes ( $\mathrm{p}<0.001$ for all combinations).

Patient characteristics with and without headache are summarized in Table 2.

The survival rates of patients with/without headaches 30 days after the stroke were $92.7 \%(127 / 137) / 98.5 \%$ (999/1014) $(\mathrm{p}<0.001)$ in CI, 84.0\% (79/94)/95.4\% $(206 / 216)(\mathrm{p}=0.002)$ in $\mathrm{CH}$, and $81.7 \%(85 / 104) / 71.4 \%$ $(5 / 7)(\mathrm{p}=0.615)$ in SAH.

Kaplan-Meier Survival Curves of patients with and without headaches are presented in Figure 1 (CI) and Figure $2(\mathrm{CH})$. The log-rank test proved significant differences in both categories $(\mathrm{p}<0.001$ and $\mathrm{p}=0.001$, respectively).

The hazard ratios for death comparing patients with and without headache are shown in Table 3.

The present study showed following associations with statistical significance;

1) Headache was most frequently seen in SAH patients, followed by $\mathrm{CH}$, and least seen in $\mathrm{CI}$, which agrees with previous reports [14].

2) Patients with headaches were younger in $\mathrm{CI}$ and in $\mathrm{CH}$. Patients with headache were younger than those without headache by 2.0 years in CI and by 3.2 years in $\mathrm{CH}$.

3) Systolic blood pressure was lower in patients without headache in SAH.

4) Diastolic blood pressure was higher in patients with headache in CI.

5) Hypertension history was more often associated with patients with headache than patients without in CI, whereas more often associated with patients without headache than patients with headache in SAH.

6) Hyperlipemia history was more often associated with patients with headache than patients without headache.

7) Tobacco use was more often associated with patients with headache in $\mathrm{CH}$.

8) Alcohol use was more often associated with patients with headache in $\mathrm{CI}$ and $\mathrm{CH}$.

9) Paresis observed more often in patients without headache than patients with headache.

10) Early mortality was higher in patients with headache than patients without headache in $\mathrm{CI}$ and $\mathrm{CH}$.

\section{DISCUSSION}

In the cohort surveyed, a few patients with SAH did not present with headache, which agrees with previous reports [15]. Naganuma et al. reported some SAH patients without headache and they found no specific clinical characteristics over patients with headache [16].

Although the difference did not reach statistically significant level, the ratio of female over male who had headache was higher than that of female who did not have headache in CI, which agrees the previous report that showed women were more likely to present with headaches [17]. The present study adds information that sex ratios of patients with/without headache differed among stroke subtypes. 
Table 2. Comparison of characteristics between patients with headache and patients without headache.

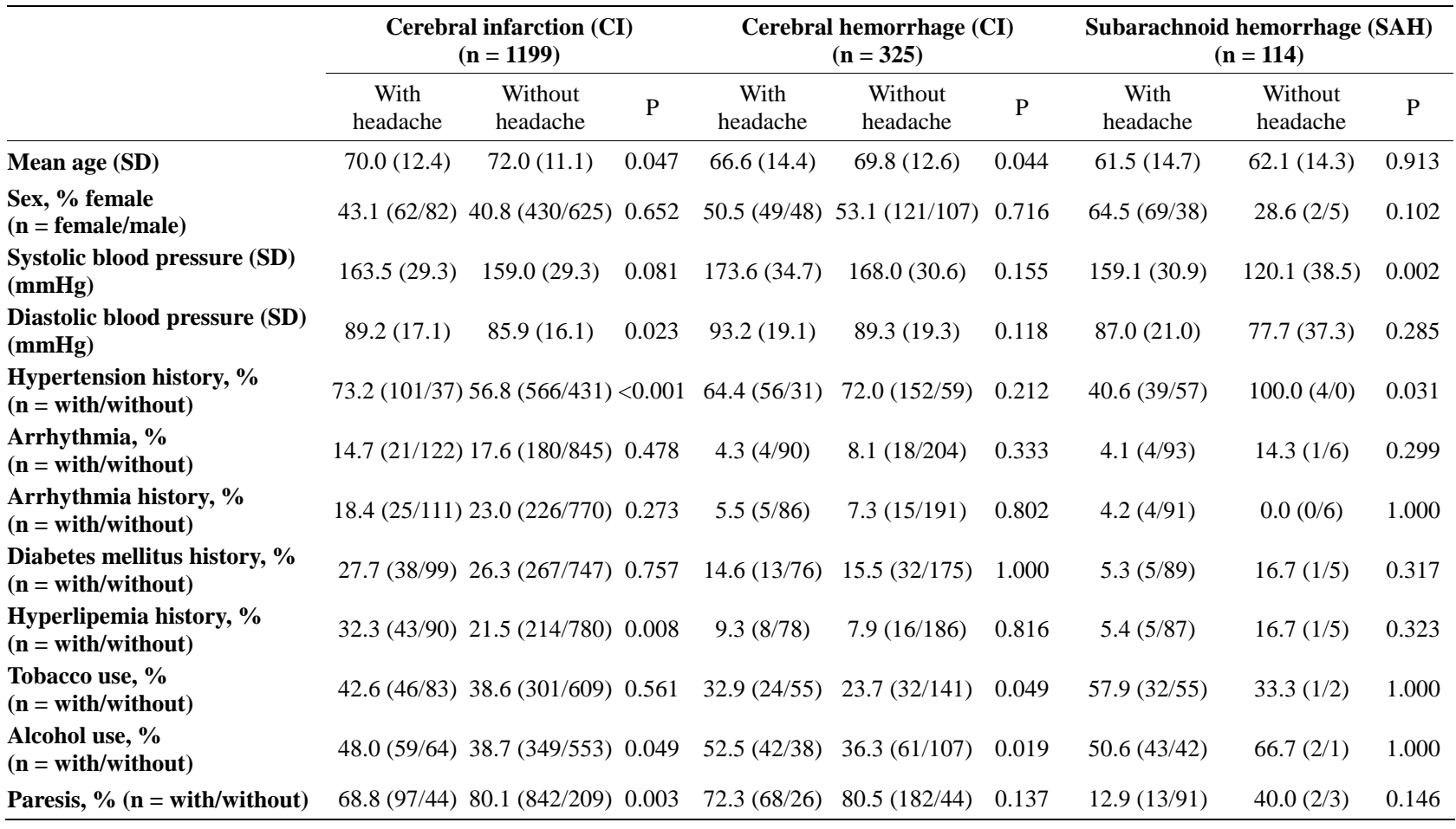

Data on some characteristics were missing in a few patients.

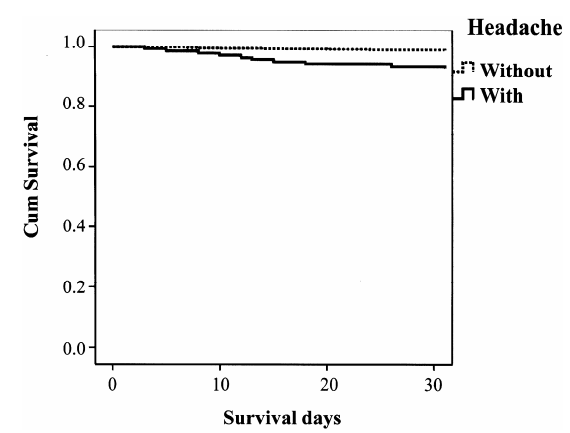

Figure 1. Kaplan-Meier survival surves of patients with cerebral infarction.

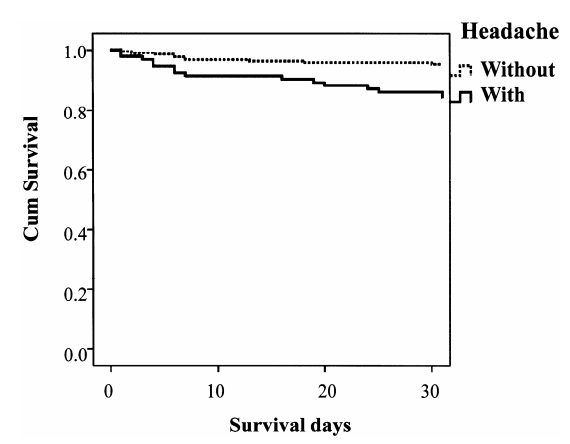

Figure 2. Kaplan-Meier survival curves of patients with cerebral hemorrhage.

Both systolic and diastolic blood pressure tended to be higher in patients with headache than patients without
Table 3. Hazard ratios for death comparing patients with headache and patients without headache (Adjusted for age, sex, systolic and diastolic blood pressure, arrhythmia, tobacco and alcohol use, and paresis).

\begin{tabular}{lccc}
\hline & Hazard ratio & $\begin{array}{c}\text { 95\% confidence } \\
\text { interval }\end{array}$ & $\mathbf{p}$ \\
\hline Overall & 5.74 & $2.94-11.23$ & $<0.001$ \\
Cerebral infarction & 4.94 & $1.73-14.08$ & 0.003 \\
Cerebral hemorrhage & 3.20 & $1.10-9.36$ & 0.033 \\
Subarachnoid hemorrhage & $1.23 \times 10^{5}$ & $0.00-$ & 0.986 \\
\hline
\end{tabular}

headache. Hypertension history was significantly higher with headache patients in CI, which agrees a previous report [18]. However, association between headache and hypertension history is still controversial $[14,19]$. Hyperlipemia history was associated with headache significantly in CI patients.

The mechanisms underlying to cause headaches in stroke are not fully understood.

Vestergaard et al. reported that 1) headache occurred in one of four acute stroke patients; 2) Unilateral headache was usually ipsilateral to the stroke lesion; 3) Headache severity was not related to the size of the ischemic stroke lesion [20]. Pain in migraine may be relevant to headache in strokes, and it has been suggested that this headache is associated with dilatation of certain arteries at the base of the brain. Most studies agree that headache may be more frequent in posterior circulation 
strokes and in dissections [21]. It is speculated that, in hemorrhage, a diffuse headache is caused by mechanical stretch and blood products that diffuse and irritate the trigeminovascular system [14]. Mitsias et al. investigated factors determining headache at onset of acute ischemic stroke, and reported three factors as the independent predictors of headache; namely, posterior circulation of the event, absence of history of hypertension and treatment with warfarin $[14,19]$. On the contrary, the present study showed hypertension history was more common and arrhythmia history was less common in patients with onset headache.

It is noteworthy that headaches were associated with mortality within 30 days after the event: the survival rates of patients with headaches were lower than those of patients without headaches by $5.8 \%$ in CI, and by $11.4 \%$ in $\mathrm{CH}$. The mortalities shown in this study cohort could be lower than those of stroke patients in general due to the exclusion criteria of the study. We excluded patients who were unable to provide reliable information on the presence or absence of onset headache mainly because of conscious disturbance.

Although headache alone does not indicate exact localization and nature of the disease, headache severity was reported not related to size of ischemic stroke lesion [20]. Leira et al. reported that headache at the onset of ischaemic stroke was an independent predictor of neurological worsening [22].

The main causes of death in the first month after stroke were neurological, followed by those related to complications of immobility, especially pneumonia [23]. At the early stage after the stroke, the leading cause of death is brain edema [24]. Stroke patients often have brain edema [25]. Two types of edema-cytotoxic and vasogenic edema-occur in patients with ischemic stroke. Vasogenic edema, characterized by fluid movement from vascular to extravascular spaces, leads to an expansion in brain volume with increased intracranial pressure, herniation, and additional ischemic injuries [26]. $\mathrm{CH}$, as well as SAH, also causes brain edema. A certain type of migraine is reported to develop with a progressive increase in cortical swelling [27]. Brain mass lesion with vasogenic edema is known to cause headache [28]. The correlation of headache with brain edema could also explain the correlation of headache with hypertension and higher mortality, which was shown in this study.

Adjusted hazard ratios for death were higher in patients with headache than in patients without in both CI and $\mathrm{CH}$. The results suggest that headache could predict death in $\mathrm{CI}$ and $\mathrm{CH}$.

\section{LIMITATIONS}

First, possible bias in assessment of headache is inevitable, since headache is an objective symptom and is essentially dependent on information from patients. Types or degree of headache are often difficult to evaluate. Some patients were unable to provide reliable information on the presence or absence of onset headache mainly because of conscious disturbance; such patients were excluded from the study.

Second, outcome depends not only on the severity of the disease, but also on treatments and patients' conditions, which were not covered in this study.

Third, inherent limitations of stroke registry of entire prefecture are difficult to be avoided. Patients with mild symptoms might not have visited hospitals and patients with atypical symptoms might not to have diagnosed as such, and subsequently might not have been registered [12].

With all limitations, significant bias which affects the major conclusions, headache at the onset of stroke correlates early mortality, is unlikely, because the study is based on large number of patients.

\section{GENERALISABILITY}

Hazard ratios for death, comparing patients with headache and patients without in SAH, didn't reach statistical significance. Patients without headache were rather exceptional in SAH. Mortality of SAH patients was much higher than those of $\mathrm{CI}$ and $\mathrm{CH}$. Therefore, headache should predict poor outcome in stroke as a whole. A high hazard ratio calculated for overall stroke patients supports the generalisability. The limitation is, however, that headache is applicable only for patients who are able to provide reliable information.

\section{CONCLUSIONS}

Headache is a common symptom in stroke patients and predicts outcome.

Headache is associated with younger age and alcohol use in $\mathrm{CI}$ and $\mathrm{CH}$. Patients who could express presence of headache at the onset of CI or $\mathrm{CH}$ have higher mortality than patients who could express absence of headache.

\section{ACKNOWLEDGEMENTS}

We acknowledge the contribution of participating institutions and their staffs who provided data in the development of the Kyoto Stroke Registry.

\section{REFERENCES}

[1] Balami, J.S., Chen, R.L., Grunwald, I.Q. and Buchan, A.M. (2011) Neurological complications of acute ischaemic stroke. Lancet Neurology, 10, 357-371. doi:10.1016/S1474-4422(10)70313-6

[2] Saita, M., Naito, T., Boku, S., Watanabe, Y., Suzuki, M., Oka, F., Takahashi, M., Sakurai, T., Sugihara, E., Haniu, 
T., Uehara, Y., Mitsuhashi, K., Fukuda, H., Isonuma, H., Lee, K. and Kobayashi, H. (2011) The efficacy of mahuang-tang (maoto) against influenza. Health, 3, 300-303. doi:10.4236/health.2011.35052

[3] Gilbert, J.W., Johnson, K.M., Larkin, G.L. and Moore, C.L. (2012) Atraumatic headache in US emergency departments: recent trends in CT/MRI utilisation and factors associated with severe intracranial pathology. Emergency Medicine Journal, 29, 576-581.

doi:10.1136/emermed-2011-200088

[4] Weng, Y.M., Weng, T.C., Lee, W.J. and Lin, H.J. (2011) Intracranial hypotension: An unusual cause of headache in the emergency department. Emergency Medicine Journal, 28, 257-258. doi:10.1136/emj.2009.079186

[5] Jorgensen, H.S., Jespersen, H.F., Nakayama, H., Raaschou, H.O. and Olsen, T.S. (1994) Headache in stroke: The Copenhagen Stroke Study. Neurology, 44, 17931797. doi:10.1212/WNL.44.10.1793

[6] Barnes, D.E., Mehta, K.M., Boscardin, W.J., Fortinsky, R.H., Palmer, R.M., Kirby, K.A. and Landefeld, C.S. (2012) Prediction of recovery, dependence or death in elders who become disabled during hospitalization. Journal of General Internal Medicine. doi:10.1007/s11606-012-2226-y

[7] Baron, J.C. (2011) Stroke: Predicting outcome after ischemic stroke-hard but achievable. Nature Reviews Neurology, 7, 253-254. doi:10.1038/nrneurol.2011.47

[8] Hermans, M.A., Leffers, P., Jansen, L.M., Keulemans, Y.C. and Stassen, P.M. (2012) The value of the Mortality in Emergency Department Sepsis (MEDS) score, C reactive protein and lactate in predicting 28-day mortality of sepsis in a Dutch emergency department. Emergency Medicine Journal, 29, 295-300. doi:10.1136/emj.2010.109090

[9] Wira III, C.R., Rivers, E., Martinez-Capolino, C., Silver, B., Iyer, G., Sherwin, R. and Lewandowski, C. (2011) Cardiac complications in acute ischemic stroke. Western Journal of Emergency Medicine, 12, 414-420. doi:10.5811/westjem.2011.2.1765

[10] Bourne, P.A. and McGrowder, D.A. (2010) Socio-demographic determinants of health status of elderly with selfreported diagnosed chronic medical conditions in Jamaica. Health, 2, 101-111. doi:10.4236/health.2010.22017

[11] Jo, S., Lee, J.B., Jin, Y.H., Jeong, T.O., Yoon, J.C., Jun, Y.K. and Park, B.Y. (2012) Modified early warning score with rapid lactate level in critically ill medical patients: The ViEWS-L score. Emergency Medicine Journal. doi:10.1136/emermed-2011-200760

[12] Shigematsu, K., Shimamura, O., Nakano, H., Watanabe, Y., Sekimoto, T., Shimizu, K., Nishizawa, A. and Makino, M. (2012) Vomiting should be a prompt predictor of stroke outcome. Emergency Medicine Journal. doi:10.1136/emermed-2012-201586

[13] Hatano, S. (1976) Experience from a multicentre stroke register: A preliminary report. Bulletin of the World Health Organization, 54, 541-553.

[14] Kumral, E., Bogousslavsky, J., Van Melle, G., Regli, F. and Pierre, P. (1995) Headache at stroke onset: The Lausanne Stroke Registry. Journal of Neurology, Neuro- surgery \& Psychiatry, 58, 490-492.

doi:10.1136/jnnp.58.4.490

[15] Adams Jr., H.P., Jergenson, D.D., Kassell, N.F. and Sahs, A.L. (1980) Pitfalls in the recognition of subarachnoid hemorrhage. JAMA, 244, 794-796. doi:10.1001/jama.1980.03310080028019

[16] Naganuma, M., Fujioka, S., Inatomi, Y., Yonehara, T., Hashimoto, Y., Hirano, T. and Uchino, M. (2008) Clinical characteristics of subarachnoid hemorrhage with or without headache. Journal of Stroke and Cerebrovascular Diseases, 17, 334-339. doi:10.1016/j.jstrokecerebrovasdis.2008.04.009

[17] Kapral, M.K., Fang, J., Hill, M.D., Silver, F., Richards, J., Jaigobin, C., Cheung, A.M. and Investigators of the Registry of the Canadian Stroke N (2005) Sex differences in stroke care and outcomes: Results from the Registry of the Canadian Stroke Network. Stroke, 36, 809-814. doi:10.1161/01.STR.0000157662.09551.e5

[18] Arboix, A., Massons, J., Oliveres, M., Arribas, M.P. and Titus, F. (1994) Headache in acute cerebrovascular disease: A prospective clinical study in 240 patients. Cephalalgia, 14, 37-40. doi:10.1046/j.1468-2982.1994.1401037.x

[19] Mitsias, P.D., Ramadan, N.M., Levine, S.R., Schultz, L. and Welch, K.M. (2006) Factors determining headache at onset of acute ischemic stroke. Cephalalgia, 26, 150-157. doi:10.1111/j.1468-2982.2005.01012.x

[20] Vestergaard, K., Andersen, G., Nielsen, M.I. and Jensen, T.S. (1993) Headache in stroke. Stroke, 24, 1621-1624. doi:10.1161/01.STR.24.11.1621

[21] Friberg, L., Olesen, J., Iversen, H.K. and Sperling, B. (1991) Migraine pain associated with middle cerebral artery dilatation: Reversal by sumatriptan. Lancet, 338, 1317. doi:10.1016/0140-6736(91)90005-A

[22] Castillo, J., Martinez, F., Leira, R., Lema, M. and Noya, M. (1994) Plasma monoamines in tension-type headache. Headache, 34, 531-535. doi:10.1111/j.1526-4610.1994.hed3409531.x

[23] Lavados, P.M., Sacks, C., Prina, L., Escobar, A., Tossi, C., Araya, F., Feuerhake, W., Galvez, M., Salinas, R. and Alvarez, G. (2005) Incidence, 30-day case-fatality rate, and prognosis of stroke in Iquique, Chile: A 2-year community-based prospective study (PISCIS project). Lancet, 365, 2206-2215. doi:10.1016/S0140-6736(05)66779-7

[24] Hacke, W., Schwab, S., Horn, M., Spranger, M., De Georgia, M. and von Kummer, R. (1996) "Malignant” middle cerebral artery territory infarction: Clinical course and prognostic signs. Archives of Neurology, 53, 309-315. doi:10.1001/archneur.1996.00550040037012

[25] Weimar, C., Mieck, T., Buchthal, J., Ehrenfeld, C.E., Schmid, E., Diener, H.C. and German Stroke Study C (2005) Neurologic worsening during the acute phase of ischemic stroke. Archives of Neurology, 62, 393-397. doi:10.1001/archneur.62.3.393

[26] Klatzo, I. (1985) Brain oedema following brain ischaemia and the influence of therapy. British Journal of Anaesthesia, 57, 18-22. doi:10.1093/bja/57.1.18

[27] Toldo, I., Cecchin, D., Sartori, S., Calderone, M., Mardari, 
R., Cattelan, F., Laverda, A.M., Drigo, P. and Battistella, P.A. (2011) Multimodal neuroimaging in a child with sporadic hemiplegic migraine: A contribution to understanding pathogenesis. Cephalalgia, 31, 751-756. doi:10.1177/0333102410392068
[28] Mullally, W.J. and Hall, K.E. (2010) Hypnic headache secondary to haemangioblastoma of the cerebellum. $\mathrm{Ce}$ phalalgia, 30, 887-889.

doi:10.1177/0333102409352911 